\title{
Fractional calculus and integral transforms of the product of a general class of polynomial and incomplete Fox-Wright functions
}

\author{
K. Jangid ${ }^{1}$, R.K. Parmar ${ }^{2 *}$ (D, R. Agarwal ${ }^{3}$ and Sunil D. Purohit ${ }^{1}$
}

\section{"Correspondence:}

rakeshparmar27@gmail.com

2Dept. of HEAS (Mathematics),

University College of Engineering

and Technology, Bikaner 334004,

Rajasthan, India

Full list of author information is

available at the end of the article

\begin{abstract}
Motivated by a recent study on certain families of the incomplete $\mathrm{H}$-functions (Srivastava et al. in Russ. J. Math. Phys. 25(1):116-138, 2018), we aim to investigate and develop several interesting properties related to product of a more general polynomial class together with incomplete Fox-Wright hypergeometric functions ${ }_{p} \Psi_{q}^{(\gamma)}(\mathfrak{t})$ and ${ }_{p} \Psi_{q}^{(\Gamma)}(\mathfrak{t})$ including Marichev-Saigo-Maeda (M-S-M) fractional integral and differential operators, which contain Saigo hypergeometric, Riemann-Liouville, and Erdélyi-Kober fractional operators as particular cases regarding different parameter selection. Furthermore, we derive several integral transforms such as Jacobi, Gegenbauer (or ultraspherical), Legendre, Laplace, Mellin, Hankel, and Euler's beta transforms.
\end{abstract}

MSC: Primary 26A33; 33C20; secondary 33C05

Keywords: Incomplete Fox-Wright functions; Fractional calculus operators; Integral transforms

\section{Introduction}

In a variety of diverse fields, together with engineering science, material science, mathematical physics, chemistry, and biology, the concept of differential equations (including fractional order) and their application have played an important role, see [5, 14, 18, 41-48]. In addition, the special functions of one or more variables are also important because they occur as solutions to these simulated differential equations. Therefore, with the development of new problems in the area of technologies in engineering and applied sciences, the subject of special functions is very diverse and is continuously growing. As a result, a number of articles on these concepts and their future implementations have been made available in the literature, see [1-4, 39, 40]. Incomplete special functions have additionally been utilized to a wide range of problems, and numerous scientific studies on incomplete special functions, along with related higher transcendental special functions, have currently been published by various authors $[6-12,15,20-23,31,35-38]$. In particular, the incomplete Fox-Wright functions ${ }_{p} \Psi_{q}^{(\gamma)}(\mathfrak{t})$ and ${ }_{p} \Psi_{q}^{(\Gamma)}(\mathfrak{t})$ with $p$ numerator and $q$ denomi-

(c) The Author(s) 2020. This article is licensed under a Creative Commons Attribution 4.0 International License, which permits use, sharing, adaptation, distribution and reproduction in any medium or format, as long as you give appropriate credit to the original author(s) and the source, provide a link to the Creative Commons licence, and indicate if changes were made. The images or other third party material in this article are included in the article's Creative Commons licence, unless indicated otherwise in a credit line to the material. If material is not included in the article's Creative Commons licence and your intended use is not permitted by statutory regulation or exceeds the permitted use, you will need to obtain permission directly from the copyright holder. To view a copy of this licence, visit http://creativecommons.org/licenses/by/4.0/. 
nator parameters are stated as follows [23, 35]:

$$
{ }_{p} \Psi_{q}^{(\gamma)}\left[\begin{array}{c}
\left(a_{1}, A_{1}, x\right),\left(a_{j}, A_{j}\right)_{2, p} ; \\
\left(b_{j}, B_{j}\right)_{1, q} ;
\end{array}\right]=\sum_{\ell=0}^{\infty} \frac{\gamma\left(a_{1}+A_{1} \ell, x\right) \prod_{j=2}^{p} \Gamma\left(a_{j}+A_{j} \ell\right)}{\prod_{j=1}^{q} \Gamma\left(b_{j}+B_{j} \ell\right)} \frac{\mathfrak{t}^{\ell}}{\ell !}
$$

and

$$
\begin{array}{r}
{ }_{p} \Psi_{q}^{(\Gamma)}\left[\begin{array}{c}
\left(a_{1}, A_{1}, x\right),\left(a_{j}, A_{j}\right)_{2, p} ; \\
\left(b_{j}, B_{j}\right)_{1, q} ;
\end{array}\right]=\sum_{\ell=0}^{\infty} \frac{\Gamma\left(a_{1}+A_{1} \ell, x\right) \prod_{j=2}^{p} \Gamma\left(a_{j}+A_{j} \ell\right)}{\prod_{j=1}^{q} \Gamma\left(b_{j}+B_{j} \ell\right)} \frac{\mathfrak{t}^{\ell}}{\ell !} \\
\left(x \geqq 0, A_{j} \in \mathbb{R}^{+}(j=1, \ldots, r) ; B_{j} \in \mathbb{R}^{+}(j=1, \ldots, s) ; 1+\sum_{j=1}^{q} B_{j}-\sum_{j=1}^{p} A_{j} \geqq 0\right),
\end{array}
$$

with

$$
|\mathfrak{t}|<\nabla:=\left(\prod_{j=1}^{p} A_{j}^{-A_{j}}\right) \cdot\left(\prod_{j=1}^{q} B_{j}^{B_{j}}\right) .
$$

The incomplete Fox-Wright functions ${ }_{p} \Psi_{q}^{(\gamma)}(\mathfrak{t})$ and ${ }_{p} \Psi_{q}^{(\Gamma)}(\mathfrak{t})$ in (1.1) and (1.2) satisfy the decomposition formula

$$
{ }_{p} \Psi_{q}^{(\gamma)}[\mathfrak{t}]+{ }_{p} \Psi_{q}^{(\Gamma)}[\mathfrak{t}]={ }_{p} \Psi_{q}[\mathfrak{t}]
$$

where ${ }_{p} \Psi_{q}[\mathfrak{t}]$ is the Fox-Wright function [16].

Also, the normalized incomplete Fox-Wright functions ${ }_{p} \Psi_{q}^{*(\gamma)}(\mathfrak{t})$ and $_{p} \Psi_{q}^{*(\Gamma)}(\mathfrak{t})$ are given by $[23,35]$

$$
{ }_{p} \Psi_{q}^{*(\gamma)}\left[\begin{array}{r}
\left(a_{1}, A_{1}, x\right),\left(a_{j}, A_{j}\right)_{2, p} ; \\
\left(b_{j}, B_{j}\right)_{1, q} ;
\end{array}\right]=\frac{\prod_{j=1}^{q} \Gamma\left(b_{j}\right)}{\prod_{j=1}^{p} \Gamma\left(a_{j}\right)} p \Psi_{q}^{(\gamma)}\left[\begin{array}{r}
\left(a_{1}, A_{1}, x\right),\left(a_{j}, A_{j}\right)_{2, p} ; \\
\left(b_{j}, B_{j}\right)_{1, q} ;
\end{array}\right]
$$

and

$$
{ }_{p} \Psi_{q}^{*(\Gamma)}\left[\begin{array}{rr}
\left(a_{1}, A_{1}, x\right),\left(a_{j}, A_{j}\right)_{2, p} ; \\
\left(b_{j}, B_{j}\right)_{1, q} ;
\end{array}\right]=\frac{\prod_{j=1}^{q} \Gamma\left(b_{j}\right)}{\prod_{j=1}^{p} \Gamma\left(a_{j}\right)}{ }^{p} \Psi_{q}^{(\Gamma)}\left[\begin{array}{r}
\left(a_{1}, A_{1}, x\right),\left(a_{j}, A_{j}\right)_{2, p} ; \\
\left(b_{j}, B_{j}\right)_{1, q} ;
\end{array}\right] .
$$

Let $S_{n}^{m}[\mathfrak{u}]$ denote the general family of polynomials made known through Srivastava [30]:

$$
S_{n}^{m}[\mathfrak{u}]=\sum_{s=0}^{[n / m]} \frac{(-n)_{m s}}{s !} A_{n, \mathfrak{u}} \mathfrak{u}^{s} \quad(n=0,1,2, \ldots)
$$

Here, $m$ is a positive integer (arbitrary), the coefficients $A_{n, s} \in \mathbb{R}$ (or $\mathbb{C}$ ) are constants (arbitrary), and $(\lambda)_{v}=\frac{\Gamma(\lambda+v)}{\Gamma(\lambda)}(\lambda, v \in \mathbb{C})$ denotes the shifted factorial (or the Pochhammer symbol). Also, the above polynomials provide a large number spectrum of well-known polynomials as one of its particular cases on appropriately specializing the coefficient $A_{n, s}$. Particularly, by setting $m=1, A_{n, s}=\frac{s !}{(-n)_{m s}}$ for $s=k$ and $A_{n, s}=0$ for $s \neq k$, the general class of polynomials leads to a power function, i.e.,

$$
S_{n}^{m}[\mathfrak{u}]=\mathfrak{u}^{k} \quad\left(k \in \mathbb{Z}^{+} \text {with } k \leq n\right) .
$$


Taking into account formula (1.3), it is also appropriate to study the characteristics and properties of the incomplete Fox-Wright function ${ }_{p} \Psi_{q}^{(\Gamma)}(\mathfrak{t})$.

\section{Fractional integral and differential operators}

We recall a general pair of fractional integral and differential operators popularly known as Marichev-Saigo-Maeda (M-S-M), which involve, in their kernel, third Appell's twovariable hypergeometric function $F_{3}($.$) and are defined by [32]$

$$
F_{3}\left(\sigma, \sigma^{\prime}, \rho, \rho^{\prime} ; \eta ; \mathfrak{X}, \mathfrak{Y}\right)=\sum_{m, n=0}^{\infty} \frac{(\sigma)_{m}\left(\sigma^{\prime}\right)_{n}(\rho)_{m}\left(\rho^{\prime}\right)_{n}}{(\eta)_{m+n}} \frac{\mathfrak{X}}{m !} \frac{\mathfrak{Y}}{n !} \quad(\max \{|\mathfrak{X}|,|\mathfrak{Y}|<1\}) .
$$

Here, we mention and study left-hand-sided fractional integral and differential operators(see, for details, $[19,26,28])$.

Definition 1 For $\sigma, \sigma^{\prime}, \rho, \rho^{\prime}, \eta \in \mathbb{C}$ and $x>0$ with $\Re(\eta)>0$, the left-hand-sided MarichevSaigo-Maeda (M-S-M) fractional integral and differential operators are respectively defined as

$$
\begin{aligned}
\left(\mathcal{I}_{0+}^{\sigma, \sigma^{\prime}, \rho, \rho^{\prime}, \eta} f\right)(\mathfrak{X})= & \frac{\mathfrak{X}^{-\sigma}}{\Gamma(\eta)} \int_{0}^{\mathfrak{X}}(\mathfrak{X}-\mathfrak{t})^{\eta-1} \mathfrak{t}^{-\sigma^{\prime}} \\
& \times F_{3}\left(\sigma, \sigma^{\prime}, \rho, \rho^{\prime} ; \eta ; 1-\frac{\mathfrak{t}}{\mathfrak{X}}, 1-\frac{\mathfrak{X}}{\mathfrak{t}}\right) f(\mathfrak{t}) \mathrm{d} \mathfrak{t}
\end{aligned}
$$

and

$$
\begin{aligned}
\left(\mathcal{D}_{0+}^{\sigma, \sigma^{\prime}, \rho, \rho^{\prime}, \eta} f\right)(\mathfrak{X})= & \left(I_{0+}^{-\sigma^{\prime},-\sigma,-\rho^{\prime},-\rho,-\eta} f\right)(\mathfrak{X}) \\
= & \left(\frac{d}{d \mathfrak{X}}\right)^{\kappa}\left(\mathcal{I}_{0+}^{-\sigma^{\prime},-\sigma,-\rho^{\prime}+[\Re(\eta)]+1,-\rho,-\eta+\kappa+1} f\right)(\mathfrak{X}) \\
= & \frac{1}{\Gamma(\kappa-\eta)}\left(\frac{d}{d \mathfrak{X}}\right)^{\kappa} \mathfrak{X}^{\sigma_{1}^{\prime}} \int_{0}^{\mathfrak{X}}(\mathfrak{X}-\mathfrak{t})^{\kappa-\eta-1} \mathfrak{t}^{\sigma} \\
& \times F_{3}\left(-\sigma^{\prime},-\sigma, \kappa-\rho^{\prime},-\rho ; \kappa-\eta ; 1-\frac{\mathfrak{t}}{\mathfrak{X}}, 1-\frac{\mathfrak{X}}{\mathfrak{t}}\right) f(\mathfrak{t}) \mathrm{d} \mathfrak{t}
\end{aligned}
$$

where $\kappa=[\Re(\eta)]+1$ and $[\Re(\eta)]$ denotes the integer part of $\Re(\eta)$.

The preceding results are well known and can be used as a proof of subsequent theorems.

Lemma Let $\sigma, \sigma^{\prime}, \rho, \rho^{\prime}, \eta, \lambda \in \mathbb{C}$ such that $\Re(\eta)>0$.

(a) If $\Re(\lambda)>\max \left\{0, \Re\left(\sigma^{\prime}-\rho^{\prime}\right), \Re\left(\sigma+\sigma^{\prime}+\rho-\eta\right)\right\}$, then

$$
\begin{aligned}
& \left(\mathcal{I}_{0+}^{\sigma, \sigma^{\prime}, \rho, \rho^{\prime}, \eta} \mathfrak{t}^{\lambda-1}\right)(\mathfrak{X}) \\
& \quad=\frac{\Gamma(\lambda) \Gamma\left(-\sigma^{\prime}+\rho^{\prime}+\lambda\right) \Gamma\left(-\sigma-\sigma^{\prime}-\rho+\eta+\lambda\right)}{\Gamma\left(\rho^{\prime}+\lambda\right) \Gamma\left(-\sigma-\sigma^{\prime}+\eta+\lambda\right) \Gamma\left(-\sigma^{\prime}-\rho+\eta+\lambda\right)} \mathfrak{X}^{-\sigma-\sigma^{\prime}+\eta+\lambda-1} .
\end{aligned}
$$


(b) If $\Re(\lambda)>\max \left\{0, \Re(-\sigma+\rho), \mathfrak{R}\left(-\sigma-\sigma^{\prime}-\rho^{\prime}+\eta\right)\right\}$, then

$$
\begin{aligned}
& \left(\mathcal{D}_{0+}^{\sigma, \sigma^{\prime}, \rho, \rho^{\prime}, \eta} \mathfrak{t}^{\lambda-1}\right)(\mathfrak{X}) \\
& \quad=\frac{\Gamma(\lambda) \Gamma(\sigma-\rho+\lambda) \Gamma\left(\sigma+\sigma^{\prime}+\rho^{\prime}-\eta+\lambda\right)}{\Gamma(-\rho+\lambda) \Gamma\left(\sigma+\sigma^{\prime}-\eta+\lambda\right) \Gamma\left(\sigma+\rho^{\prime}-\eta+\lambda\right)} \mathfrak{X}^{\sigma+\sigma^{\prime}-\eta+\lambda-1} .
\end{aligned}
$$

Firstly, we shall investigate the left-hand-sided Marichev-Saigo-Maeda (M-S-M) fractional integral and differential operators of the product of a general family of polynomial along with incomplete Fox-Wright functions ${ }_{p} \Psi_{q}^{(\Gamma)}(\mathfrak{t})$.

Theorem 1 Let $\sigma, \sigma^{\prime}, \rho, \rho^{\prime}, \eta, \lambda \in \mathbb{C}$ be such that $\Re(\eta), \mu, v>0$ and $\Re(\lambda)>\max \{\Re(-s \mu)$, $\left.\mathfrak{R}\left(\sigma^{\prime}-\rho^{\prime}-s \mu\right), \mathfrak{R}\left(\sigma+\sigma^{\prime}+\rho-\eta-s \mu\right)\right\}$. Thereupon, for $\mathfrak{X}>0$,

$$
\begin{aligned}
& \left(\mathcal{I}_{0+}^{\sigma, \sigma^{\prime}, \rho, \rho^{\prime}, \eta}\left(\mathfrak{t}^{\lambda-1} S_{n}^{m}\left[\mathfrak{t}^{\mu}\right]_{p} \Psi_{q}^{(\Gamma)}\left[\mathfrak{t}^{\nu}\right]\right)\right)(\mathfrak{X}) \\
& =\mathfrak{X}^{\lambda-\sigma-\sigma^{\prime}+\eta-1} \sum_{s=0}^{[n / m]} \frac{(-n)_{m s}}{s !} A_{n, s} \mathfrak{X}^{s \mu} \\
& \times{ }_{p+3} \Psi_{q+3}^{(\Gamma)}\left[\begin{array}{c}
\left(a_{1}, A_{1}, x\right),(\lambda+s \mu, v),\left(-\sigma^{\prime}+\rho^{\prime}+\lambda+s \mu, v\right), \\
\left(\rho^{\prime}+\lambda+s \mu, v\right),\left(-\sigma-\sigma^{\prime}+\eta+\lambda+s \mu, v\right),
\end{array}\right. \\
& \left.\begin{array}{cc}
\left(-\sigma-\sigma^{\prime}-\rho+\eta+\lambda+s \mu, v\right),\left(a_{j}, A_{j}\right)_{2, p} ; & \mathfrak{X}^{v} \\
\left(-\sigma^{\prime}-\rho+\eta+\lambda+s \mu, v\right),\left(b_{j}, B_{j}\right)_{1, q} ; &
\end{array}\right] .
\end{aligned}
$$

Proof For the sake of simplicity, let us consider

$$
\mathfrak{L}=\left(\mathcal{I}_{0+}^{\sigma, \sigma^{\prime}, \rho, \rho^{\prime}, \eta}\left(\mathfrak{t}^{\lambda-1} S_{n}^{m}\left[\mathfrak{t}^{\mu}\right]_{p} \Psi_{q}^{(\Gamma)}\left[\mathfrak{t}^{\nu}\right]\right)\right)(\mathfrak{X})
$$

Now, using (1.2) and (1.6) in (2.7) and then taking advantage of relationship (2.4), we find for $\mathfrak{X}>0$

$$
\begin{aligned}
\mathfrak{L}= & \left(\mathcal{I}_{0+}^{\sigma, \sigma^{\prime}, \rho, \rho^{\prime}, \eta}\left(\mathfrak{t}^{\lambda-1} \sum_{s=0}^{[n / m]} \frac{(-n)_{m s}}{s !} A_{n, t^{s}}{ }^{s \mu} \sum_{\ell=0}^{\infty} \frac{\Gamma\left(a_{1}+A_{1} \ell, x\right) \prod_{j=2}^{p} \Gamma\left(a_{j}+A_{j} \ell\right)}{\prod_{j=1}^{q} \Gamma\left(b_{j}+B_{j} \ell\right)} \frac{\mathfrak{t}^{\ell v}}{\ell !}\right)\right)(\mathfrak{X}) \\
= & \sum_{s=0}^{[n / m]} \frac{(-n)_{m s}}{s !} A_{n, s} \sum_{\ell=0}^{\infty} \frac{\Gamma\left(a_{1}+A_{1} \ell, x\right) \prod_{j=2}^{p} \Gamma\left(a_{j}+A_{j} \ell\right)}{\prod_{j=1}^{q} \Gamma\left(b_{j}+B_{j} \ell\right) \ell !} \times\left(\mathcal{I}_{0+}^{\sigma, \sigma^{\prime}, \rho, \rho^{\prime}, \eta}\left\{\mathfrak{t}^{\lambda+s \mu+\ell \nu-1}\right\}\right)(\mathfrak{X}) \\
= & \sum_{s=0}^{[n / m]} \frac{(-n)_{m s}}{s !} A_{n, s} \sum_{\ell=0}^{\infty} \frac{\Gamma\left(a_{1}+A_{1} \ell, x\right) \prod_{j=2}^{p} \Gamma\left(a_{j}+A_{j} \ell\right)}{\prod_{j=1}^{q} \Gamma\left(b_{j}+B_{j} \ell\right) \ell !} \times \mathfrak{X}^{-\sigma-\sigma^{\prime}+\eta+\lambda+s \mu+\ell \nu-1} \\
& \times \frac{\Gamma(\lambda+s \mu+\ell v) \Gamma\left(-\sigma^{\prime}+\rho^{\prime}+\lambda+s \mu+\ell v\right) \Gamma\left(-\sigma-\sigma^{\prime}-\rho+\eta+\lambda+s \mu+\ell \nu\right)}{\Gamma\left(\rho^{\prime}+\lambda+s \mu+\ell \nu\right) \Gamma\left(-\sigma-\sigma^{\prime}+\eta+\lambda+s \mu+\ell v\right) \Gamma\left(-\sigma^{\prime}-\rho+\eta+\lambda+s \mu+\ell \nu\right)} .
\end{aligned}
$$

Finally, in opinion of (1.2) interpretation, we get (2.6) as a desired outcome. 
Theorem 2 Let $\sigma, \sigma^{\prime}, \rho, \rho^{\prime}, \eta, \lambda, c \in \mathbb{C}$ be such that $\mu, \nu>0$ and $\Re(\lambda)>\max \{\Re(-s \mu), \Re(-\sigma+$ $\left.\rho-s \mu), \mathfrak{R}\left(-\sigma-\sigma^{\prime}-\rho^{\prime}+\eta-s \mu\right)\right\}$. Then, for $\mathfrak{X}>0$,

$$
\begin{aligned}
& \left(\mathcal{D}_{0+}^{\sigma, \sigma^{\prime}, \rho, \rho^{\prime}, \eta}\left(\mathfrak{t}^{\lambda-1} S_{n}^{m}\left[\mathfrak{t}^{\mu}\right]_{p} \Psi_{q}^{(\Gamma)}\left[\mathfrak{t}^{\nu}\right]\right)\right)(\mathfrak{X}) \\
& =\mathfrak{X}^{\lambda+\sigma+\sigma^{\prime}-\eta-1} \sum_{s=0}^{[n / m]} \frac{(-n)_{m s}}{s !} A_{n, s} \mathfrak{X}^{s \mu} \\
& \times{ }_{p+3} \Psi_{q+3}^{(\Gamma)}\left[\begin{array}{l}
\left(a_{1}, A_{1}, x\right),(\lambda+s \mu, v),(\sigma-\rho+\lambda+s \mu, v), \\
(-\rho+\lambda+s \mu, v),\left(\sigma+\sigma^{\prime}-\eta+\lambda+s \mu, v\right),
\end{array}\right. \\
& \left.\left(\sigma+\sigma^{\prime}+\rho^{\prime}-\eta+\lambda+s \mu, v\right),\left(a_{j}, A_{j}\right)_{2, p} ; \quad \mathfrak{X}^{v}\right] . \\
& \left(\sigma+\rho^{\prime}-\eta+\lambda+s \mu, v\right),\left(b_{j}, B_{j}\right)_{1, q} ;
\end{aligned}
$$

Proof For the sake of simplicity, let us consider

$$
\mathfrak{T}=\left(\mathcal{D}_{0+}^{\sigma, \sigma^{\prime}, \rho, \rho^{\prime}, \eta}\left(\mathfrak{t}^{\lambda-1} S_{n}^{m}\left[\mathfrak{t}^{\mu}\right]_{p} \Psi_{q}^{(\Gamma)}\left[\mathfrak{t}^{\nu}\right]\right)\right)(\mathfrak{X})
$$

Now using (1.2) and (1.6) in (2.9) and then taking advantage of relationship (2.5), for $\mathfrak{X}>0$, we acquire

$$
\begin{aligned}
\mathfrak{T}= & \left(\mathcal{D}_{0+}^{\sigma, \sigma^{\prime}, \rho, \rho^{\prime}, \eta}\left(\mathfrak{t}^{\lambda-1} \sum_{s=0}^{[n / m]} \frac{(-n)_{m s}}{s !} A_{n, s} s^{s \mu} \sum_{\ell=0}^{\infty} \frac{\Gamma\left(a_{1}+A_{1} \ell, x\right) \prod_{j=2}^{p} \Gamma\left(a_{j}+A_{j} \ell\right)}{\prod_{j=1}^{q} \Gamma\left(b_{j}+B_{j} \ell\right)} \frac{\mathfrak{t}^{\ell v}}{\ell !}\right)\right)(\mathfrak{X}) \\
= & \sum_{s=0}^{[n / m]} \frac{(-n)_{m s}}{s !} A_{n, s} \sum_{\ell=0}^{\infty} \frac{\Gamma\left(a_{1}+A_{1} \ell, x\right) \prod_{j=2}^{p} \Gamma\left(a_{j}+A_{j} \ell\right)}{\prod_{j=1}^{q} \Gamma\left(b_{j}+B_{j} \ell\right) \ell !} \times\left(\mathcal{D}_{0+}^{\sigma, \sigma^{\prime}, \rho, \rho^{\prime}, \eta}\left\{\mathfrak{t}^{\lambda+s \mu+\ell \nu-1}\right\}\right)(\mathfrak{X}) \\
= & \sum_{s=0}^{[n / m]} \frac{(-n)_{m s}}{s !} A_{n, s} \sum_{\ell=0}^{\infty} \frac{\Gamma\left(a_{1}+A_{1} \ell, x\right) \prod_{j=2}^{p} \Gamma\left(a_{j}+A_{j} \ell\right)}{\prod_{j=1}^{q} \Gamma\left(b_{j}+B_{j} \ell\right) \ell !} \times \mathfrak{X}^{\sigma+\sigma^{\prime}-\eta+\lambda+s \mu+\ell \nu-1} \\
& \times \frac{\Gamma(\lambda+s \mu+\ell v) \Gamma\left(\sigma+\sigma^{\prime}+\rho^{\prime}-\eta+\lambda+s \mu+\ell v\right) \Gamma(\sigma-\rho+\lambda+s \mu+\ell \nu)}{\Gamma(-\rho+\lambda+s \mu+\ell \nu) \Gamma\left(\sigma+\rho^{\prime}-\eta+\lambda+s \mu+\ell \nu\right) \Gamma\left(\sigma+\sigma^{\prime}-\eta+\lambda+s \mu+\ell \nu\right)} .
\end{aligned}
$$

Finally, in opinion of the (1.2) interpretation, we get (2.8) as a desired outcome.

The $F_{3}$ Appell function in (2.1) tends to reduce to the hypergeometric function ${ }_{2} F_{1}$ of Gauss as follows:

$$
\begin{aligned}
{ }_{2} F_{1}(\sigma, \rho ; \eta ; \mathfrak{X}) & =F_{3}\left(\sigma, \sigma^{\prime}, \rho, \rho^{\prime} ; \eta ; \mathfrak{X}, 0\right) \\
& =F_{3}\left(\sigma, 0, \rho, \rho^{\prime} ; \eta ; \mathfrak{X}, \mathfrak{Y}\right) \\
& =F_{3}\left(\sigma, \sigma^{\prime}, \rho, 0 ; \eta ; \mathfrak{X}, \mathfrak{Y}\right) .
\end{aligned}
$$

In the light of the reduction formula (2.10), the Marichev-Saigo-Maeda operators (2.2) including (2.3) reduce to Saigo's hypergeometric fractional operators. If we take $\sigma=\sigma+\rho$, $\sigma^{\prime}=\rho^{\prime}=0, \rho=-\eta, \eta=\sigma$, we immediately obtain Saigo's fractional integral along with differential operators in conjunction with the hypergeometric function ${ }_{2} F_{1}[26,27]$ :

$$
\left(\mathcal{I}_{0,+}^{\sigma, \rho, \eta} f\right)(\mathfrak{X})=\frac{\mathfrak{X}^{-\sigma-\rho}}{\Gamma(\sigma)} \int_{0}^{\mathfrak{X}}(\mathfrak{X}-\mathfrak{t})^{\sigma-1}{ }_{2} F_{1}\left(\sigma+\rho,-\eta ; \sigma ; 1-\frac{\mathfrak{t}}{\mathfrak{X}}\right) f(\mathfrak{t}) \mathrm{d} \mathfrak{t},
$$


and

$$
\begin{aligned}
\left(\mathcal{D}_{0,+}^{\sigma, \rho, \eta} f\right)(\mathfrak{X}) & =\left(\mathcal{I}_{0,+}^{-\sigma,-\rho, \sigma+\eta} f\right)(\mathfrak{X}) \\
& =\left(\frac{d}{d \mathfrak{X}}\right)^{\kappa}\left(\mathcal{I}_{0,+}^{-\sigma+\kappa,-\rho-\kappa, \sigma+\eta-\kappa} f\right)(\mathfrak{X}) \quad(\kappa=[\mathfrak{R}(\sigma)]+1) .
\end{aligned}
$$

Furthermore, by specializing the parameters in (2.11) and (2.12), we obtain RiemannLiouville and Erdélyi-Kober fractional operators. Setting $\rho=-\sigma$ in (2.11) and (2.12) yields the familiar Riemann-Liouville integrals and derivatives of fractional order $\sigma \in \mathbb{C}$ beside $\Re(\sigma)>0$ as well as $\mathfrak{X} \in \mathbb{R}^{+}$(see, e.g., [17]):

$$
\left(\mathcal{I}_{0,+}^{\sigma,-\sigma, \eta} f\right)(\mathfrak{X})=\left(\mathcal{I}_{0,+}^{\sigma} f\right)(\mathfrak{X}) \equiv \frac{1}{\Gamma(\sigma)} \int_{0}^{\mathfrak{X}}(\mathfrak{X}-\mathfrak{t})^{\sigma-1} f(\mathfrak{t}) \mathrm{d} \mathfrak{t}
$$

and

$$
\begin{aligned}
\left(\mathcal{D}_{0,+}^{\sigma,-\sigma, \eta} f\right)(\mathfrak{X}) & =\left(\mathcal{D}_{0,+}^{\sigma} f\right)(\mathfrak{X}) \\
& =\left(\frac{d}{d \mathfrak{X}}\right)^{\kappa}\left(\mathcal{I}_{0,+}^{\kappa-\sigma} f\right)(\mathfrak{X}) \quad(\kappa=[\Re(\sigma)]+1) .
\end{aligned}
$$

Again setting $\rho=0$ in (2.11) and (2.12) provides the so-called Erdélyi-Kober integrals and derivatives of fractional order $\sigma \in \mathbb{C}$ along with $\Re(\sigma)>0$ and $\mathfrak{X} \in \mathbb{R}^{+}$(see, e.g., [34]):

$$
\left(\mathcal{I}_{0,+}^{\sigma, 0, \eta} f\right)(\mathfrak{X})=\left(\mathcal{I}_{\eta, \sigma}^{+} f\right)(\mathfrak{X})=\frac{\mathfrak{X}^{-\sigma-\eta}}{\Gamma(\sigma)} \int_{0}^{\mathfrak{X}}(\mathfrak{X}-\mathfrak{t})^{\sigma-1} \mathfrak{t}^{\eta} f(\mathfrak{t}) \mathrm{d} \mathfrak{t}
$$

and

$$
\begin{aligned}
\left(\mathcal{D}_{0,+}^{\sigma, 0, \eta} f\right)(\mathfrak{X}) & =\left(\mathcal{D}_{\eta, \sigma}^{+} f\right)(\mathfrak{X}) \\
& =\left(\frac{d}{d \mathfrak{X}}\right)^{n}\left(\mathcal{I}_{0,+}^{-\sigma+\kappa,-\sigma, \sigma+\eta-\kappa} f\right)(\mathfrak{X}) \quad(\kappa=[\Re(\sigma)]+1) .
\end{aligned}
$$

Here, we mention these results in form of Corollaries 2.1 to 2.6.

Corollary 2.1 Let $\sigma, \rho, \eta, \lambda \in \mathbb{C}$ be such that $\Re(\sigma), \mu, v>0$ and $\Re(\lambda)>\max \{\Re(-s \mu), \Re(\rho-$ $\eta-s \mu)$ \}. Thereupon, for $\mathfrak{X}>0$,

$$
\begin{aligned}
& \left(\mathcal{I}_{0+}^{\sigma, \rho, \eta}\left(\mathfrak{t}^{\lambda-1} S_{n}^{m}\left[\mathfrak{t}^{\mu}\right]_{p} \Psi_{q}^{(\Gamma)}\left[\mathfrak{t}^{\nu}\right]\right)\right)(\mathfrak{X}) \\
& =\mathfrak{X}^{-\rho+\lambda-1} \sum_{s=0}^{[n / m]} \frac{(-n)_{m s}}{s !} A_{n, s} \mathfrak{X}^{s \mu} \\
& \quad \times{ }_{p+2} \Psi_{q+2}^{(\Gamma)}\left[\begin{array}{c}
\left.\left(a_{1}, A_{1}, x\right),(\lambda+s \mu, v),(-\rho+\eta+\lambda+s \mu, v),\left(a_{j}, A_{j}\right)_{2, p} ; \quad \mathfrak{X}^{v}\right] . \\
(\sigma+\eta+\lambda+s \mu, v),(-\rho+\lambda+s \mu, v),\left(b_{j}, B_{j}\right)_{1, q} ;
\end{array}\right]
\end{aligned}
$$


Corollary 2.2 Suppose $\sigma, \eta, \lambda \in \mathbb{C}$ to be such that $\Re(\sigma), \mu, v>0$ and $\Re(\lambda)>\max \{\Re(-s \mu)$, $\mathfrak{i}(-\sigma-\eta-s \mu)\}$. Thereupon, for $\mathfrak{X}>0$,

$$
\begin{aligned}
& \left(\mathcal{I}_{0+}^{\sigma,-\sigma, \eta}\left(\mathfrak{t}^{\lambda-1} S_{n}^{m}\left[\mathfrak{t}^{\mu}\right]_{p} \Psi_{q}^{(\Gamma)}\left[\mathfrak{t}^{\nu}\right]\right)\right)(\mathfrak{X}) \\
& =\mathfrak{X}^{\sigma+\lambda-1} \sum_{s=0}^{[n / m]} \frac{(-n)_{m s}}{s !} A_{n, s} \mathfrak{X}^{s \mu} \\
& \times_{p+1} \Psi_{q+1}^{(\Gamma)}\left[\begin{array}{c}
\left(a_{1}, A_{1}, x\right),(\lambda+s \mu, v),\left(a_{j}, A_{j}\right)_{2, p} ; \\
(\sigma+\lambda+s \mu, v),\left(b_{j}, B_{j}\right)_{1, q} ;
\end{array} \quad \mathfrak{X}^{v}\right] .
\end{aligned}
$$

Corollary 2.3 Let $\sigma, \eta, \lambda \in \mathbb{C}$ be such that $\Re(\sigma), \mu, v>0$ and $\Re(\lambda)>\max \{\Re(-s \mu), \Re(-\eta-$ $s \mu)\}$. Thereupon, for $\mathfrak{X}>0$,

$$
\begin{aligned}
\left(\mathcal{I}_{\eta, \sigma}^{+}\left(\mathfrak{t}^{\lambda-1} S_{n}^{m}\left[\mathfrak{t}^{\mu}\right]_{p} \Psi_{q}^{(\Gamma)}\left[\mathfrak{t}^{\nu}\right]\right)\right)(\mathfrak{X}) & \\
= & \mathfrak{X}^{\lambda-1} \sum_{s=0}^{[n / m]} \frac{(-n)_{m s}}{s !} A_{n, s} \mathfrak{X}^{s \mu} \\
& \times{ }_{p+1} \Psi_{q+1}^{(\Gamma)}\left[\begin{array}{c}
\left(a_{1}, A_{1}, x\right),(\eta+\lambda+s \mu, v),\left(a_{j}, A_{j}\right)_{2, p} ; \\
(\sigma+\eta+\lambda+s \mu, v),\left(b_{j}, B_{j}\right)_{1, q} ;
\end{array}\right] .
\end{aligned}
$$

Corollary 2.4 Let $\sigma, \rho, \eta, \lambda \in \mathbb{C}$ be such that $\mu, v>0$ and $\mathfrak{R}(\lambda)>\max \{\Re(-s \mu), \Re(-\sigma-\rho-$ $\eta-s \mu)\}$. Then, for $\mathfrak{X}>0$,

$$
\begin{aligned}
& \left(\mathcal{D}_{0+}^{\sigma, \rho, \eta}\left(\mathfrak{t}^{\lambda-1} S_{n}^{m}\left[\mathfrak{t}^{\mu}\right]_{p} \Psi_{q}^{(\Gamma)}\left[\mathfrak{t}^{\nu}\right]\right)\right)(\mathfrak{X}) \\
& =\mathfrak{X}^{\rho+\lambda-1} \sum_{s=0}^{[n / m]} \frac{(-n)_{m s}}{s !} A_{n, s} \mathfrak{X}^{s \mu} \\
& \quad \times{ }_{p+2} \Psi_{q+2}^{(\Gamma)}\left[\begin{array}{c}
\left(a_{1}, A_{1}, x\right),(\lambda+s \mu, \nu),(\sigma+\rho+\eta+\lambda+s \mu, v),\left(a_{j}, A_{j}\right)_{2, p} ; \\
(\eta+\lambda+s \mu, v),(\rho+\lambda+s \mu, v),\left(b_{j}, B_{j}\right)_{1, q} ;
\end{array}\right] .
\end{aligned}
$$

Corollary 2.5 Let $\sigma, \eta, \lambda \in \mathbb{C}$ be such that $\mu, v>0$ and $\Re(\lambda)>\max \{\Re(-s \mu), \Re(-\eta-s \mu)\}$. Then, for $\mathfrak{X}>0$,

$$
\begin{aligned}
& \left(\mathcal{D}_{0+}^{\sigma,-\sigma, \eta}\left(\mathfrak{t}^{\lambda-1} S_{n}^{m}\left[\mathfrak{t}^{\mu}\right]_{p} \Psi_{q}^{(\Gamma)}\left[\mathfrak{t}^{\nu}\right]\right)\right)(\mathfrak{X}) \\
& =\mathfrak{X}^{-\sigma+\lambda-1} \sum_{s=0}^{[n / m]} \frac{(-n)_{m s}}{s !} A_{n, s} \mathfrak{X}^{s \mu} \\
& \quad \times{ }_{p+1} \Psi_{q+1}^{(\Gamma)}\left[\begin{array}{c}
\left(a_{1}, A_{1}, x\right),(\lambda+s \mu, v),\left(a_{j}, A_{j}\right)_{2, p} ; \\
(-\sigma+\lambda+s \mu, v),\left(b_{j}, B_{j}\right)_{1, q} ;
\end{array}\right] .
\end{aligned}
$$


Corollary 2.6 Let $\sigma, \eta, \lambda \in \mathbb{C}$ be such that $\mu, v>0$ and $\Re(\lambda)>\max \{\Re(-s \mu), \Re(-\sigma-\eta-$ $s \mu)\}$. Thereupon, for $\mathfrak{X}>0$,

$$
\begin{aligned}
\left(\mathcal{D}_{\eta, \sigma}^{+}\left(\mathfrak{t}^{\lambda-1} S_{n}^{m}\left[\mathfrak{t}^{\mu}\right]_{p} \Psi_{q}^{(\Gamma)}\left[\mathfrak{t}^{\nu}\right]\right)\right)(\mathfrak{X}) & \\
= & \mathfrak{X}^{\lambda-1} \sum_{s=0}^{[n / m]} \frac{(-n)_{m s}}{s !} A_{n, s} \mathfrak{X}^{s \mu} \\
& \times{ }_{p+1} \Psi_{q+1}^{(\Gamma)}\left[\begin{array}{c}
\left(a_{1}, A_{1}, x\right),(\sigma+\eta+\lambda+s \mu, v),\left(a_{j}, A_{j}\right)_{2, p} ; \\
(\eta+\lambda+s \mu, v),\left(b_{j}, B_{j}\right)_{1, q} ;
\end{array}\right.
\end{aligned}
$$

\section{Integral transforms}

In this part, several integral transforms such as Jacobi, Gegenbauer (or ultraspherical), Legendre, Laplace, Mellin, Hankel, and Euler's beta transforms of a product of a general polynomial class and incomplete Fox-Wright function ${ }_{p} \Psi_{q}^{(\Gamma)}(\mathfrak{t})$ are presented.

\subsection{Jacobi and related integral transforms}

The classical orthogonal Jacobi polynomial $P_{n}^{(\mathfrak{h}, \mathfrak{g})}(\mathfrak{t})$ is given by the following (see, for example, [33]):

$$
P_{n}^{(\mathfrak{h}, \mathfrak{g})}(\mathfrak{t})=(-1)^{n}(-\mathfrak{t})=\left(\begin{array}{c}
\mathfrak{h}+n \\
n
\end{array}\right){ }_{2} F_{1}\left[\begin{array}{c|c}
-n, \mathfrak{h}+\mathfrak{g}+n+1 & \frac{1-\mathfrak{t}}{2} \\
\mathfrak{h}+1 &
\end{array}\right.
$$

where ${ }_{2} F_{1}$ is the Gauss hypergeometric function [25].

Definition 2 The Jacobi transformation of a $f(\mathfrak{t})$ function is set as follows (see, e.g., [13, p. 501]):

$$
\begin{gathered}
\mathbb{J}^{(\mathfrak{h}, \mathfrak{g})}[f(\mathfrak{t}) ; n]=\int_{-1}^{1}(1-\mathfrak{t})^{\mathfrak{h}}(1+\mathfrak{t})^{\mathfrak{g}} P_{n}^{(\mathfrak{h}, \mathfrak{g})}(\mathfrak{t}) f(\mathfrak{t}) \mathrm{d} \mathfrak{t} \\
\left(\min \{\mathfrak{R}(\mathfrak{h}), \Re(\mathfrak{g})\}>-1 ; n \in \mathbb{N}_{0}\right),
\end{gathered}
$$

provided that the $f(\mathfrak{t})$ function seems to be so limited that only the integral exists in (3.2).

The Jacobi transform of the power function $t^{\rho-1}$ is given by (see, e.g., [37, p. 128, Eq. (18)])

$$
\begin{aligned}
\mathbb{J}^{(\mathfrak{h}, \mathfrak{g})}\left[\mathfrak{t}^{\rho-1} ; n\right]= & \int_{-1}^{1}(1-\mathfrak{t})^{\xi-1}(1+\mathfrak{t})^{\eta-1} P_{n}^{(\mathfrak{h}, \mathfrak{g})}(\mathfrak{t}) \mathfrak{t}^{\rho-1} \mathrm{~d} \mathfrak{t} \\
= & 2^{\xi+\eta-1}\left(\begin{array}{c}
\mathfrak{h}+n \\
n
\end{array}\right) \mathrm{B}(\xi, \eta) F_{1: 1 ; 0}^{1: 2 ; 1}\left[\begin{array}{ll}
\xi:-n, \mathfrak{h}+\mathfrak{g}+n+1 ; 1-\rho ; \\
\xi+\eta: \quad \mathfrak{h}+1 ; \quad ; \quad 1,2
\end{array}\right] \\
& \left(\min \{\mathfrak{R}(\xi), \Re(\eta)\}>0 ; \rho \in \mathbb{C} ; n \in \mathbb{N}_{0}\right),
\end{aligned}
$$

where $F_{p: l ; \mu}^{q: m ; v}$ corresponds to Kampé de Fériet's function in two variables (see, e.g., [32, p. 22, Eq. 1.3(2)] and [32, p. 37, Eq. 1.4(21)]). In fact, this last integral formula (3.3) will be 
reduced instantly to the preceding form when specifying $\xi=\mathfrak{h}+1$ and $\eta=\mathfrak{g}+1$ :

$$
\begin{aligned}
\mathbb{J}^{(\mathfrak{h}, \mathfrak{g})}\left[\mathfrak{t}^{\rho-1} ; \mathfrak{n}\right]= & \int_{-1}^{1}(1-\mathfrak{t})^{\mathfrak{h}}(1+\mathfrak{t})^{\mathfrak{g}} P_{n}^{(\mathfrak{h}, \mathfrak{g})}(\mathfrak{t}) \mathfrak{t}^{\rho-1} \mathrm{~d} \mathfrak{t} \\
= & 2^{\mathfrak{h}+\mathfrak{g}+1}\left(\begin{array}{c}
\mathfrak{h}+n \\
n
\end{array}\right) \mathrm{B}(\mathfrak{h}+1, \mathfrak{g}+1) \\
& \times F_{1: 1 ; 0}^{1: 2 ; 1}\left[\begin{array}{ccc}
\mathfrak{h}+1:-n, \mathfrak{h}+\mathfrak{g}+n+1 ; 1-\rho ; & 1,2 \\
\mathfrak{h}+\mathfrak{g}+2: \quad \mathfrak{h}+1 ; \quad-;
\end{array}\right] \\
& \left(\min \{\mathfrak{R}(\mathfrak{h}), \mathfrak{R}(\mathfrak{g})\}>-1 ; \rho \in \mathbb{C} ; n \in \mathbb{N}_{0}\right) .
\end{aligned}
$$

However, in its additional limited case where $\rho=m+1\left(m \in \mathbb{N}_{0}\right)$, (3.4) brings the established consequence about the $\mathfrak{t}^{m}\left(m \in \mathbb{N}_{0}\right)$ Jacobi transform studied by [25, p. 261, Eq. (14) along with (15)]:

$$
\begin{aligned}
\mathbb{J}^{(\mathfrak{h}, \mathfrak{g})} & {\left[\mathfrak{t}^{m} ; n\right] } \\
= & \int_{-1}^{1}(1-\mathfrak{t})^{\mathfrak{h}}(1+\mathfrak{t})^{\mathfrak{g}} P_{n}^{(\mathfrak{h}, \mathfrak{g})}(\mathfrak{t}) \mathfrak{t}^{m} \mathrm{~d} \mathfrak{t} \\
= & \begin{cases}0 & (m=0,1,2, \ldots, n-1), \\
2^{\mathfrak{h}+\mathfrak{g}+n+1} \mathrm{~B}(\mathfrak{h}+n+1, \mathfrak{g}+n+1) & (m=n), \\
2^{\mathfrak{h}+\mathfrak{g}+n+1}\left(\begin{array}{c}
m \\
n
\end{array}\right) \mathrm{B}(\mathfrak{h}+n+1, \mathfrak{g}+n+1) & \\
\cdot{ }_{2} F_{1}\left[\begin{array}{l}
n-m, \mathfrak{h}+n+1 \\
\mathfrak{h}+\mathfrak{g}+2 n+2
\end{array} \mid 2\right], & (m=n+1, n+2, n+3, \ldots)\end{cases} \\
& \left(\min \{\mathfrak{R}(\mathfrak{h}), \mathfrak{R}(\mathfrak{g})\}>-1 ; m, n \in \mathbb{N}_{0}\right) .
\end{aligned}
$$

Specifying the parameters $\mathfrak{h}$ and $\mathfrak{g}$, the Jacobi polynomials $P_{n}^{(\mathfrak{h}, \mathfrak{g})}(\mathfrak{t})$ exhibit, like in their individual cases, other such recognized orthogonal polynomials being the Gegenbauer (or ultraspherical) polynomials $C_{n}^{v}(\mathfrak{t})$, the Legendre (or spherical) polynomials $P_{n}(\mathfrak{t}$ ), and the Tchebycheff polynomials $T_{n}(\mathfrak{t})$ and $U_{n}(\mathfrak{t})$ of the first kind and second kind (see, for details, [33]). In addition, we have the accompanying established connections with the Gegenbauer polynomials $C_{n}^{v}(\mathfrak{t})$ as well as the Legendre polynomials $P_{n}(\mathfrak{t})$ :

$$
C_{n}^{v}(\mathfrak{t})=\left(\begin{array}{c}
v+n-\frac{1}{2} \\
n
\end{array}\right)^{-1}\left(\begin{array}{c}
2 v+n-1 \\
n
\end{array}\right) P_{n}^{\left(v-\frac{1}{2}, v-\frac{1}{2}\right)}(\mathfrak{t})
$$

and

$$
P_{n}(\mathfrak{t})=C_{n}^{\frac{1}{2}}(\mathfrak{t})=P_{n}^{(0,0)}(\mathfrak{t}),
$$

respectively, which, in conjunction with (3.2), brings the Gegenbauer transform $\mathbb{G}^{(v)}[f(\mathfrak{t})$; $n]$ as follows:

$$
\begin{aligned}
\mathbb{G}^{(v)} & {[f(\mathfrak{t}) ; n] } \\
& =\left(\begin{array}{c}
v+n-\frac{1}{2} \\
n
\end{array}\right)^{-1}\left(\begin{array}{c}
2 v+n-1 \\
n
\end{array}\right) \mathbb{J}^{\left(v-\frac{1}{2}, v-\frac{1}{2}\right)}[f(\mathfrak{t}) ; n] \\
& =\int_{-1}^{1}\left(1-\mathfrak{t}^{2}\right)^{v-\frac{1}{2}} C_{n}^{v}(\mathfrak{t}) f(\mathfrak{t}) \mathrm{d} \mathfrak{t} \quad\left(\Re(v)>-\frac{1}{2} ; n \in \mathbb{N}_{0}\right),
\end{aligned}
$$


and the resulting Legendre transform $\mathbb{L}[f(\mathfrak{t}) ; n]$ which is described by

$$
\mathbb{L}[f(\mathfrak{t}) ; n]=\mathbb{G}^{\left(\frac{1}{2}\right)}[f(\mathfrak{t}) ; n]=\int_{-1}^{1} P_{n}(\mathfrak{t}) f(\mathfrak{t}) \mathrm{d} \mathfrak{t} \quad\left(n \in \mathbb{N}_{0}\right)
$$

We are now generating three new results that provide the relations between Jacobi, Gegenbauer, and Legendre transforms with the following incomplete Fox-Wright function ${ }_{p} \Psi_{q}^{(\Gamma)}$ (1.2).

Theorem 3 The preceding formula for Jacobi transform is valid under the condition stated in (1.2):

$$
\begin{aligned}
\mathbb{J}^{(\mathfrak{h}, \mathfrak{g})} & \left\{\mathfrak{t}^{\rho-1} S_{n^{\prime}}^{m^{\prime}}[\omega \mathfrak{t}]_{p} \Psi_{q}^{(\Gamma)}[\omega \mathfrak{t}] ; n\right\} \\
= & 2^{\mathfrak{h}+\mathfrak{g}+1}\left(\begin{array}{c}
\mathfrak{h}+n \\
n
\end{array}\right) \mathrm{B}(\mathfrak{h}+1, \mathfrak{g}+1) \\
& \times \sum_{s=0}^{\left[n^{\prime} / m^{\prime}\right]} \frac{\left(-n^{\prime}\right)_{m^{\prime} s}}{s !} A_{n^{\prime}, s} \omega^{s} \sum_{\ell=0}^{\infty} \frac{\Gamma\left(a_{1}+A_{1} \ell, x\right) \prod_{j=2}^{p} \Gamma\left(a_{j}+A_{j} \ell\right)}{\prod_{j=1}^{q} \Gamma\left(b_{j}+B_{j} \ell\right)} \\
& \times F_{1: 1 ; 0}^{1: 2 ; 1}\left[\begin{array}{c}
\mathfrak{h}+1:-n, \mathfrak{h}+\mathfrak{g}+n+1 ; 1-\rho-s-\ell ; \quad 1,2] \frac{\omega^{\ell}}{\ell !} \\
\mathfrak{h}+\mathfrak{g}+2: \quad \mathfrak{h}+1 ; \quad-;
\end{array}\right. \\
& \left(x \geqq 0 ; n \in \mathbb{N}_{0} ; \min \{\mathfrak{R}(\mathfrak{h}), \mathfrak{R}(\mathfrak{g})\}>-1 ; \rho \in \mathbb{C} ; p, q \in \mathbb{N}_{0}\right),
\end{aligned}
$$

where the Jacobi transform into (3.4) is assumed to exist.

Proof By employing the concept of (3.2) together with (1.2), we get

$$
\begin{aligned}
\mathbb{J}^{(\mathfrak{h}, \mathfrak{g})} & \left\{\mathfrak{t}^{\rho-1} S_{n^{\prime}}^{m^{\prime}}[\omega \mathfrak{t}]_{p} \Psi_{q}^{(\Gamma)}[\omega \mathfrak{t}] ; n\right\} \\
= & \int_{-1}^{1} \mathfrak{t}^{\rho-1}(1-\mathfrak{t})^{\mathfrak{h}}(1+\mathfrak{t})^{\mathfrak{g}} P_{n}^{(\mathfrak{h}, \mathfrak{g})}(\mathfrak{t}) S_{n^{\prime}}^{m^{\prime}}[\omega \mathfrak{t}]_{p} \Psi_{q}^{(\Gamma)}[\omega \mathfrak{t}] \mathrm{dt} \\
= & \int_{-1}^{1} \mathfrak{t}^{\rho-1}(1-\mathfrak{t})^{\mathfrak{h}}(1+\mathfrak{t})^{\mathfrak{g}} P_{n}^{(\mathfrak{h}, \mathfrak{g})}(\mathfrak{t}) \sum_{s=0}^{\left[n^{\prime} / m^{\prime}\right]} \frac{\left(-n^{\prime}\right)_{m^{\prime} s}}{s !} A_{n^{\prime}, s}(\omega \mathfrak{t})^{s} \\
& \times \sum_{\ell=0}^{\infty} \frac{\Gamma\left(a_{1}+A_{1} \ell, x\right) \prod_{j=2}^{p} \Gamma\left(a_{j}+A_{j} \ell\right)}{\prod_{j=1}^{q} \Gamma\left(b_{j}+B_{j} \ell\right)} \frac{(\omega \mathfrak{t})^{\ell}}{\ell !} \mathrm{dt},
\end{aligned}
$$

where, when adjusting the order of integration and summation (that might be easily explained by absolute convergence), we make use of the Jacobi transform formula (3.4) along with the parameter $\rho$ substituted by $\rho+k\left(\rho \in \mathbb{C} ; k \in \mathbb{N}_{0}\right)$.

By employing the Jacobi transform formula (3.5), we can simplify the assertion (3.10) of Theorem 3 in their limiting case when $\rho=m+1\left(m \in \mathbb{N}_{0}\right)$. Furthermore, in light 
of connection (3.6), Theorem 3 gives the subsequent corollary by considering $\mathfrak{h}=\mathfrak{g}=$ $v-\frac{1}{2}$.

Corollary 3.1 The following Gegenbauer transform formula holds true under the condition stated in (1.2):

$$
\begin{aligned}
\mathbb{G}^{(v)}[ & {\left[\mathfrak{t}^{\rho-1} S_{n^{\prime}}^{m^{\prime}}[\omega \mathfrak{t}]_{p} \Psi_{q}^{(\Gamma)}[\omega \mathfrak{t}] ; n\right] } \\
= & 2^{2 v}\left(\begin{array}{c}
2 v+n-1 \\
n
\end{array}\right) \mathrm{B}\left(v+\frac{1}{2}, v+\frac{1}{2}\right) \\
& \times \sum_{s=0}^{\left[n^{\prime} / m^{\prime}\right]} \frac{\left(-n^{\prime}\right)_{m^{\prime} s}}{s !} A_{n^{\prime}, s} \omega^{s} \sum_{\ell=0}^{\infty} \frac{\Gamma\left(a_{1}+A_{1} \ell, x\right) \prod_{j=2}^{p} \Gamma\left(a_{j}+A_{j} \ell\right)}{\prod_{j=1}^{q} \Gamma\left(b_{j}+B_{j} \ell\right)} \\
& \times F_{1: 1 ; 0}^{1: 2 ; 1}\left[\begin{array}{l}
v+\frac{1}{2}:-n, 2 v+n ; 1-\rho-\ell ; \\
2 v+1: \quad v+\frac{1}{2} ; \quad-;
\end{array}\right] \frac{\omega^{\ell}}{\ell !} \\
& \left(x \geqq 0 ; n \in \mathbb{N}_{0} ; \rho \in \mathbb{C} ; p, q \in \mathbb{N}_{0}\right),
\end{aligned}
$$

where it is assumed that the Gegenbauer transform in (3.12) exists.

A special case of Theorem 3 when $\mathfrak{h}=\mathfrak{g}=0$ (or, alternatively, Corollary 3.1 with $v=\frac{1}{2}$ ) gives the following result for the Legendre transform described by (3.9).

Corollary 3.2 The subsequent Legendre transform formula holds true under the condition stated in (1.2):

$$
\begin{aligned}
\mathbb{L}\left[\mathfrak{t}^{\rho-1} S_{n^{\prime}}^{m^{\prime}}[\omega \mathfrak{t}]_{p} \Psi_{q}^{(\Gamma)}[\omega \mathfrak{t}] ; n\right] & =2 \sum_{s=0}^{\left[n^{\prime} / m^{\prime}\right]} \frac{\left(-n^{\prime}\right)_{m^{\prime} s}}{s !} A_{n^{\prime}, s} \omega^{s} \sum_{\ell=0}^{\infty} \frac{\Gamma\left(a_{1}+A_{1} \ell, x\right) \prod_{j=2}^{p} \Gamma\left(a_{j}+A_{j} \ell\right)}{\prod_{j=1}^{q} \Gamma\left(b_{j}+B_{j} \ell\right)} \\
& \times F_{1: 1 ; 0}^{1: 2 ; 1}\left[\begin{array}{cc}
1:-n, n+1 ; 1-\rho-\ell ; & 1,2] \frac{\omega^{\ell}}{\ell !} \\
2: \quad 1 ; \quad-; & 1, \quad
\end{array}\right. \\
& \left(x \geqq 0 ; n \in \mathbb{N}_{0} ; \rho \in \mathbb{C} ; p, q \in \mathbb{N}_{0}\right),
\end{aligned}
$$

where it is assumed that the Legendre transform in (3.13) exists.

\subsection{Laplace transform}

The Laplace transform of a given function $f(\mathfrak{t})$ is defined as follows [13, 29]:

$$
\mathrm{L}\{f(\mathfrak{t}) ; \omega\}=\int_{0}^{\infty} e^{-\omega \mathfrak{t}} f(\mathfrak{t}) \mathrm{dt} \quad(\Re(\omega)>0)
$$

if the improper integral exists. 
Theorem 4 If $\Delta>0, \alpha>0, \beta>0$, and $\Re(\omega)>0$, then the Laplace transform of incomplete Fox-Wright function ${ }_{p} \Psi_{q}^{(\Gamma)}$ is given as follows:

$$
\begin{aligned}
& \mathrm{L}\left\{\mathfrak{t}^{\lambda-1} S_{n}^{m}\left[\mathfrak{t}^{\alpha}\right]_{p} \Psi_{q}^{(\Gamma)}\left[\mathfrak{t}^{\beta}\right] ; \omega\right\} \\
& =\frac{1}{\omega^{\lambda}} \sum_{s=0}^{[n / m]} \frac{(-n)_{m s}}{s !} A_{n, s} \frac{1}{\omega^{s \alpha}}
\end{aligned}
$$

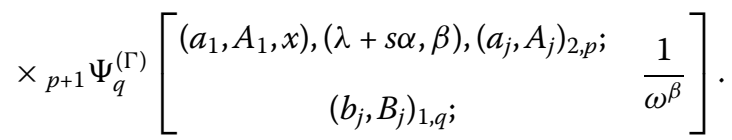

Proof Using the definition of (1.2) and (1.6) in the left-hand side of (3.15) and applying the definition of Laplace transform (3.14), we obtain

$$
\begin{aligned}
& \mathrm{L}\left\{\mathfrak{t}^{\lambda-1} S_{n}^{m}\left[\mathfrak{t}^{\alpha}\right]_{p} \Psi_{q}^{(\Gamma)}\left[\mathfrak{t}^{\beta}\right] ; \omega\right\} \\
& \quad=\mathrm{L}\left\{\mathfrak{t}^{\lambda-1} \sum_{s=0}^{[n / m]} \frac{(-n)_{m s}}{s !} A_{n, s} s^{s \alpha} \sum_{\ell=0}^{\infty} \frac{\Gamma\left(a_{1}+A_{1} \ell, x\right) \prod_{j=2}^{p} \Gamma\left(a_{j}+A_{j} \ell\right)}{\prod_{j=1}^{q} \Gamma\left(b_{j}+B_{j} \ell\right)} \frac{\mathfrak{t}^{\ell \beta}}{\ell !} ; \omega\right\} \\
& \quad=\sum_{s=0}^{[n / m]} \frac{(-n)_{m s}}{s !} A_{n, s} \sum_{\ell=0}^{\infty} \frac{\Gamma\left(a_{1}+A_{1} \ell, x\right) \prod_{j=2}^{p} \Gamma\left(a_{j}+A_{j} \ell\right)}{\prod_{j=1}^{q} \Gamma\left(b_{j}+B_{j} \ell\right) \ell !} \int_{0}^{\infty} e^{-\omega \mathfrak{t} \mathfrak{t}^{\lambda+s \alpha+\ell \beta-1} \mathrm{~d} \mathfrak{t}} \\
& =\sum_{s=0}^{[n / m]} \frac{(-n)_{m s}}{s !} A_{n, s} \sum_{\ell=0}^{\infty} \frac{\Gamma\left(a_{1}+A_{1} \ell, x\right) \prod_{j=2}^{p} \Gamma\left(a_{j}+A_{j} \ell\right)}{\prod_{j=1}^{q} \Gamma\left(b_{j}+B_{j} \ell\right) \ell !} \frac{\Gamma(\lambda+s \alpha+\ell \beta)}{\omega^{\lambda+s \alpha+\ell \beta}} .
\end{aligned}
$$

Now, employing the gamma function formula [25]

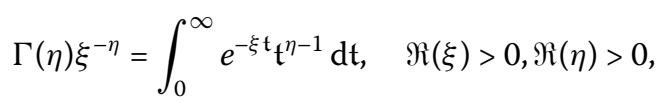

and then using (1.2), we arrive at the desired result in Theorem 4.

\subsection{Mellin transform}

The Mellin transform of a given function $f(\mathfrak{t})$ is represented as follows [13, 29]:

$$
\mathrm{M}\{f(\mathfrak{t}) ; \omega\}=\int_{0}^{\infty} \mathfrak{t}^{\omega-1} f(\mathfrak{t}) \mathrm{d} \mathfrak{t} \quad(\Re(\omega)>0),
$$

given that the improper integral exists.

Theorem 5 If $\Delta>0, \mathfrak{h}>0, \mathfrak{g}>0, \alpha>0, \beta>0$, and $\mathfrak{R}(\omega)>0$, then the Mellin transform of incomplete Fox-Wright function ${ }_{p} \Psi_{q}^{(\Gamma)}$ is given as follows:

$$
\begin{aligned}
M & \left\{S_{n}^{m}\left[\frac{\mathfrak{h}}{(1+\mathfrak{t})^{\alpha}}\right] p \Psi_{q}^{(\Gamma)}\left[\frac{\mathfrak{g}}{(1+\mathfrak{t})^{\beta}}\right] ; \omega\right\} \\
= & \Gamma(\omega) \sum_{s=0}^{[n / m]} \frac{(-n)_{m s}}{s !} A_{n, s} \mathfrak{h}^{s} \\
& \times_{p+1} \Psi_{q+1}^{(\Gamma)}\left[\begin{array}{cc}
\left(a_{1}, A_{1}, x\right),(-\omega+s \alpha, \beta),\left(a_{j}, A_{j}\right)_{2, p} ; & \mathfrak{g} \\
(s \alpha, \beta),\left(b_{j}, B_{j}\right)_{1, q} ;
\end{array}\right.
\end{aligned}
$$


Proof Using the definition of (1.2), (1.6) and applying Mellin transform (3.17) in the lefthand side of (3.18) and then employing the beta function formula [25]

$$
B(\alpha, \beta)= \begin{cases}\int_{0}^{\infty} \frac{\mathrm{t}^{\alpha-1}}{(1-\mathrm{t})} \mathrm{d}^{\alpha+\beta} \mathrm{dt} & (\Re(\alpha)>0 ; \Re(\beta)>0), \\ \frac{\Gamma(\alpha) \Gamma(\beta)}{\Gamma(\alpha+\beta)} & \left(\alpha, \beta \notin \mathbb{Z}_{0}^{-}\right),\end{cases}
$$

after using definition (1.2), we obtain the desired formula in Theorem 5.

\subsection{Hankel transform}

The Hankel transform of a given function $f(\mathfrak{t})$ is characterized as follows [13, 29]:

$$
\mathrm{H}_{v}\{f(\mathfrak{t}) ; \omega\}=\int_{0}^{\infty} \mathfrak{t} J_{v}(\omega \mathfrak{t}) f(\mathfrak{t}) \mathrm{dt} \quad(\Re(\omega)>0)
$$

provided that the improper integral exists, $J_{v}(\omega t)$ is the Bessel function of order $v$.

Theorem 6 If $\Delta>0, \alpha>0, \beta>0, \lambda>0$, and $\Re(\omega)>0$, then the Hankel transform of incomplete Fox-Wright function ${ }_{p} \Psi_{q}^{(\Gamma)}$ is given as follows:

$$
\begin{aligned}
\mathrm{H}_{\nu}\left\{\mathfrak{t}^{\lambda-1} S_{n}^{m}\left[\mathfrak{t}^{\alpha}\right]_{p} \Psi_{q}^{(\Gamma)}\left[\mathfrak{t}^{\beta}\right] ; \omega\right\} \\
=\frac{2}{\omega^{\lambda}} \sum_{s=0}^{[n / m]} \frac{(-n)_{m s}}{s !} A_{n, s}\left(\frac{2}{\omega}\right)^{s \alpha} \\
\quad \times{ }_{p+1} \Psi_{q+1}^{(\Gamma)}\left[\begin{array}{c}
\left.\left(a_{1}, A_{1}, x\right),\left(\frac{\lambda+\nu+s \alpha}{2}, \frac{\beta}{2}\right),\left(a_{j}, A_{j}\right)_{2, p} ; \quad\left(\frac{2}{\omega}\right)^{\beta}\right] . \\
\left(\frac{2+\nu-\lambda-s \alpha}{2},-\frac{\beta}{2}\right),\left(b_{j}, B_{j}\right)_{1, q} ;
\end{array}\right.
\end{aligned}
$$

Proof Using the definition of (1.2), (1.6) and applying Hankel transform (3.20) in the lefthand side of (3.21) and then employing the formula (Prudnikov, Brychkov, and Marichev $[24,(2.44)])$

$$
\int_{0}^{\infty} t^{\lambda-1} J_{\nu}(a t) \mathrm{d} t=2^{\lambda-1} a^{-\lambda} \frac{\Gamma\left(\frac{\lambda+v}{2}\right)}{\Gamma\left(1+\frac{v-\lambda}{2}\right)} \quad(a>0,-\Re(v)<\Re(\lambda)<3 / 2),
$$

we are led easily to the right-hand side of the assertion (3.21) of Theorem 6. The details are omitted here.

\subsection{Euler's beta transform}

The integral transform of Euler's beta type for a given function $f(\mathfrak{t})$ is characterized as follows $[13,29]$ :

$$
\mathrm{B}\{f(\mathfrak{t}): \alpha, \beta\}=\int_{0}^{1} \mathfrak{t}^{\alpha-1}(1-\mathfrak{t})^{\beta-1} f(\mathfrak{t}) \mathrm{dt} \quad(\Re(\alpha)>0, \Re(\beta)>0) .
$$


Theorem 7 If $\Delta>0, \mathfrak{h}>0, \mathfrak{g}>0, \mu>0, v>0, \xi>0, \eta>0$, and $\Re(\alpha), \Re(\beta)>0$, then Euler's beta type transform of incomplete Fox-Wright function ${ }_{p} \Psi_{q}^{(\Gamma)}$ is given as follows:

$$
\begin{aligned}
\mathrm{B}\left\{S_{n}^{m}\left[\mathfrak{h} \mathfrak{t}^{\mu}(1-\mathfrak{t})^{\nu}\right]_{p} \Psi_{q}^{(\Gamma)}\left[\mathfrak{g} \mathfrak{t}^{\xi}(1-\mathfrak{t})^{\eta}\right]: \alpha, \beta\right\} & \\
= & \sum_{s=0}^{[n / m]} \frac{(-n)_{m s}}{s !} A_{n, s} \mathfrak{h}^{s} \\
& \times{ }_{p+2} \Psi_{q+1}^{(\Gamma)}\left[\begin{array}{cc}
\left(a_{1}, A_{1}, x\right),(\alpha+s \mu, \xi),(\beta+s v, \eta),\left(a_{j}, A_{j}\right)_{2, p} ; & \mathfrak{g} \\
(\alpha+\beta+s(\mu+\nu), \xi+\eta),\left(b_{j}, B_{j}\right)_{1, q} ; &
\end{array}\right] .
\end{aligned}
$$

Proof Using the definition of (1.2), (1.6) and applying Euler's beta transform (3.23) in the left-hand side of (3.24) and then employing the beta function formula [25]

$$
B(\alpha, \beta)= \begin{cases}\int_{0}^{1} \mathfrak{t}^{\alpha-1}(1-\mathfrak{t})^{\beta-1} \mathrm{~d} \mathfrak{t} & (\Re(\alpha)>0 ; \Re(\beta)>0), \\ \frac{\Gamma(\alpha) \Gamma(\beta)}{\Gamma(\alpha+\beta)} & \left(\alpha, \beta \notin \mathbb{Z}_{0}^{-}\right),\end{cases}
$$

after using definition (1.2), we arrive at the desired formula in Theorem 7.

\section{Concluding remarks and observations}

In our present study, with the aid of the incomplete Fox-Wright functions ${ }_{p} \Psi_{q}^{(\gamma)}(\mathfrak{t})$ and ${ }_{p} \Psi_{q}^{(\Gamma)}(\mathfrak{t})$, we have studied several interesting properties, such as Marichev-Saigo-Maeda left-handed fractional integral and differential operators, which include Saigo hypergeometric fractional integral and differential operators, Riemann-Liouville, and ErdélyiKober fractional integral and differential operators as particular cases for various choices of parameter. In a similar pattern, one can derive results for right-hand-sided MarichevSaigo-Maeda, Saigo hypergeometric fractional, Riemann-Liouville, and Erdélyi-Kober fractional integral and differential operators. Furthermore, we derive several integral transforms such as Jacobi, Gegenbauer (or ultraspherical), Legendre, Laplace, Mellin, Hankel, and Euler's beta transforms. Specific cases of derived findings can be developed by suitably specializing the coefficient $A_{n, s}$ to obtain a large number of spectrum of the known polynomials (see, e.g. [30]). Here we give three main results, and we left the remaining ones for interested readers. If we set $n=0$ and $A_{0,0}$ (the polynomial family $S_{0}^{m}$ will reduce to unity) in Theorems 1, 2, and 3, we get the following corollaries.

Corollary 7.1 Let $\sigma, \sigma^{\prime}, \rho, \rho^{\prime}, \eta, \lambda \in \mathbb{C}$ be such that $\Re(\eta), \mu, v>0$ and $\Re(\lambda)>\max \left\{0, \Re\left(\sigma^{\prime}-\right.\right.$ $\left.\left.\rho^{\prime}\right), \Re\left(\sigma+\sigma^{\prime}+\rho-\eta\right)\right\}$. Thereupon, for $\mathfrak{X}>0$,

$$
\begin{aligned}
\left(\mathcal{I}_{0+}^{\sigma, \sigma^{\prime}, \rho, \rho^{\prime}, \eta}\left(\mathfrak{t}^{\lambda-1}{ }_{p} \Psi_{q}^{(\Gamma)}\left[\mathfrak{t}^{\nu}\right]\right)\right)(\mathfrak{X}) & \\
= & \mathfrak{X}_{p+3}^{\lambda-\sigma-\sigma^{\prime}+\eta-1} \Psi_{q+3}^{(\Gamma)}\left[\begin{array}{c}
\left(a_{1}, A_{1}, x\right),(\lambda, \nu),\left(-\sigma^{\prime}+\rho^{\prime}+\lambda, \nu\right), \\
\left(\rho^{\prime}+\lambda, \nu\right),\left(-\sigma-\sigma^{\prime}+\eta+\lambda, v\right),
\end{array}\right. \\
& \left(-\sigma-\sigma^{\prime}-\rho+\eta+\lambda, v\right),\left(a_{j}, A_{j}\right)_{2, p} ; \\
\quad & \left.\mathfrak{X}^{\nu}\right] .
\end{aligned}
$$


Corollary 7.2 Let $\sigma, \sigma^{\prime}, \rho, \rho^{\prime}, \eta, \lambda, c \in \mathbb{C}$ be such that $\mu, v>0$ and $\Re(\lambda)>\max \{0, \Re(-\sigma+$ $\left.\rho), \Re\left(-\sigma-\sigma^{\prime}-\rho^{\prime}+\eta\right)\right\}$. Then, for $\mathfrak{X}>0$,

$$
\begin{aligned}
& \left(\mathcal{D}_{0+}^{\sigma, \sigma^{\prime}, \rho, \rho^{\prime}, \eta}\left(\mathfrak{t}^{\lambda-1}{ }_{p} \Psi_{q}^{(\Gamma)}\left[\mathfrak{t}^{\nu}\right]\right)\right)(\mathfrak{X}) \\
& =\mathfrak{X}^{\lambda+\sigma+\sigma^{\prime}-\eta-1}{ }_{p+3} \Psi_{q+3}^{(\Gamma)}\left[\begin{array}{l}
\left(a_{1}, A_{1}, x\right),(\lambda, v),(\sigma-\rho+\lambda, v), \\
(-\rho+\lambda, \nu),\left(\sigma+\sigma^{\prime}-\eta+\lambda, \nu\right),
\end{array}\right. \\
& \left.\begin{array}{c}
\left(\sigma+\sigma^{\prime}+\rho^{\prime}-\eta+\lambda, \nu\right),\left(a_{j}, A_{j}\right)_{2, p} ; \quad \mathfrak{X}^{\nu} \\
\left(\sigma+\rho^{\prime}-\eta+\lambda, \nu\right),\left(b_{j}, B_{j}\right)_{1, q} ;
\end{array}\right] .
\end{aligned}
$$

Corollary 7.3 The coming Jacobi transform formula holds true under the condition stated in (1.2):

$$
\begin{aligned}
\mathbb{J}^{(\mathfrak{h}, \mathfrak{g})}\left\{\mathfrak{t}_{p}^{\rho-1} \Psi_{q}^{(\Gamma)}[\omega \mathfrak{t}] ; n\right\} \\
=2^{\mathfrak{h + g}+1}\left(\begin{array}{c}
\mathfrak{h}+n \\
n
\end{array}\right) \mathrm{B}(\mathfrak{h}+1, \mathfrak{g}+1) \sum_{\ell=0}^{\infty} \frac{\Gamma\left(a_{1}+A_{1} \ell, x\right) \prod_{j=2}^{p} \Gamma\left(a_{j}+A_{j} \ell\right)}{\prod_{j=1}^{q} \Gamma\left(b_{j}+B_{j} \ell\right)} \\
\quad \times F_{1: 1 ; 1 ; 0}^{1: 2 ;}\left[\begin{array}{c}
\mathfrak{h}+1:-n, \mathfrak{h}+\mathfrak{g}+n+1 ; 1-\rho-\ell ; \quad 1,2] \frac{\omega^{\ell}}{\ell !} \\
\mathfrak{h}+\mathfrak{g}+2: \quad \mathfrak{h}+1 ; \quad ; \quad
\end{array}\right. \\
\quad\left(x \geqq 0 ; n \in \mathbb{N}_{0} ; \min \{\mathfrak{R}(\mathfrak{h}), \mathfrak{R}(\mathfrak{g})\}>-1 ; \rho \in \mathbb{C} ; p, q \in \mathbb{N}_{0}\right),
\end{aligned}
$$

where it is considered that the Jacobi transform in (3.4) exists.

Remark 1 It is important to note that the particular cases of the results obtained in this paper for $x=0$ would give the corresponding new results for the product of a more general class of polynomials and Fox-Wright hypergeometric functions ${ }_{p} \Psi_{q}(\mathfrak{t})$.

\section{Acknowledgements}

This work is carried out under the Competitive Research Scheme (CRS) by the TEQIP-III (ATU) Rajasthan Technical University Kota under sanction number TEQIP-III/RTU(ATU)CRS/2019-20/50.

Funding

Not applicable.

Availability of data and materials

Not applicable.

Competing interests

The authors declare that they have no competing interests.

\section{Authors' contributions}

The authors contributed equally and significantly in writing this paper. All authors read and approved the final manuscript.

\section{Author details}

'Dept. of HEAS (Mathematics), Rajasthan Technical University, Kota, Rajasthan, India. ${ }^{2}$ Dept. of HEAS (Mathematics), University College of Engineering and Technology, Bikaner 334004, Rajasthan, India. ${ }^{3}$ Dept. of Mathematics, Malaviya National Institute of Technology, Jaipur 302017, Rajasthan, India.

\section{Publisher's Note}

Springer Nature remains neutral with regard to jurisdictional claims in published maps and institutional affiliations. 


\section{References}

1. Al-Omari, S.K.Q.: Some estimate of a generalized Bessel-Struve transform on certain space of generalized functions. Ukr. Math. J. 69(9), 1155-1165 (2017)

2. Al-Omari, S.K.Q.: On a class of generalized functions for some integral transform enfolding kernels of Meijer G function type. Commun. Korean Math. Soc. 33(2), 515-525 (2018)

3. Al-Omari, S.K.Q.: Estimation of a modified integral associated with a special function kernel of Fox's $H$-function type. Commun. Korean Math. Soc. 35(1), 125-136 (2020)

4. Al-Omari, S.K.Q., Jumah, G., Al-Omari, J., Saxena, D.: A new version of the generalized Krätzel-Fox integral operators. Mathematics 6, 222 (2018)

5. Cao, Y., Ma, W.-G., Ma, L.-C.: Local fractional functional method for solving diffusion equations on Cantor sets. Abstr. Appl. Anal. 2014, 803693 (2014)

6. Çetinkaya, A.: The incomplete second Appell hypergeometric functions. Appl. Math. Comput. 219, $8332-8337$ (2013)

7. Chaudhry, M.A., Qadir, A.: Incomplete exponential and hypergeometric functions with applications to non-central $\chi^{2}$-distribution. Commun. Stat., Theory Methods 34, 525-535 (2002)

8. Choi, J., Parmar, R.K.: The incomplete Lauricella and fourth Appell functions. Far East J. Math. Sci. 96, 315-328 (2015)

9. Choi, J., Parmar, R.K.: The incomplete Srivastava's triple hypergeometric functions $\gamma_{A}^{H}$ and $\Gamma_{A}^{H}$. Miskolc Math. Notes 19(1), 191-200 (2017)

10. Choi, J., Parmar, R.K., Chopra, P.: The incomplete Lauricella and first Appell functions and associated properties. Honam Math. J. 36, 531-542 (2014)

11. Choi, J., Parmar, R.K., Chopra, P.: The incomplete Srivastava's triple hypergeometric functions $\gamma_{B}^{H}$ and $\Gamma_{B}^{H}$. Filomat 30 , 1779-1787 (2016)

12. Choi, J., Parmar, R.K., Srivastava, H.M.: The incomplete Lauricella functions of several variables and associated properties and formulas. Kyungpook Math. J. 58(1), 19-35 (2018)

13. Debnath, L., Bhatta, D.: Integral Transforms and Their Applications, 3rd edn. Chapman \& Hall, London (2014)

14. Gao, F.: General fractional calculus in non-singular power-law kernel applied to model anomalous diffusion phenomena in heat transfer problems. Therm. Sci. 21(suppl. 1), S11-S18 (2017)

15. Jangid, K., Bhatter, S., Meena, S., Baleanu, D., Qurashi, M.A., Purohit, S.D.: Some fractional calculus findings associated with the incomplete I-functions. Adv. Differ. Equ. 2020, 265 (2020)

16. Kilbas, A.A., Saigo, M., Trujillo, J.J.: On the generalized Wright function. Fract. Calc. Appl. Anal. 5, $431-460$ (2002)

17. Kiryakova, V.: Generalized Fractional Calculus and Applications. Pitman Research Notes in Mathematics Series, vol. 301. Longman, Harlow (1994). Copublished in the United States with Wiley, New York

18. Liu, J.-G., Yang, X.-J., Feng, Y.-Y., Zhang, H.-Y.: On the generalized time fractional diffusion equation: symmetry analysis, conservation laws, optimal system and exact solutions. Int. J. Geom. Methods Mod. Phys. 17(1), 2050013 (2020)

19. Marichev, O.I.: Volterra equation of Mellin convolution type with a Horn function in the kernel. IzV. AN BSSR Ser Fiz.-Mat. Nauk. 1, 128-129 (1974) [in Russian]

20. Meena, S., Bhatter, S., Jangid, K., Purohit, S.D.: Some expansion formulas for incomplete $H$ and $\bar{H}$-functions involving Bessel functions. Adv. Differ. Equ. 2020, 562 (2020)

21. Meena, S., Bhatter, S., Jangid, K., Purohit, S.D.: Certain integral transforms concerning the product of family of polynomials and generalized incomplete functions. Moroccan J. Pure Appl. Anal. 6(2), 243-254 (2020)

22. Parmar, R.K., Saxena, R.K.: The incomplete generalized $\tau$-hypergeometric and second $\tau$-Appell functions. J. Korean Math. Soc. 53, 363-379 (2016)

23. Parmar, R.K., Saxena, R.K.: Incomplete extended Hurwitz-Lerch zeta functions and associated properties. Commun. Korean Math. Soc. 32, 287-304 (2017)

24. Prudnikov, A.P., Brychkov, Y.A., Marichev, O.I.: Integrals and Series, Vol. 3, More Special Functions. Gordon \& Breach, New York (1990)

25. Rainville, E.D.: Special Functions. Macmillan, New York (1960). Reprinted by Chelsea, Bronx, New York, 1971

26. Saigo, M.: On generalized fractional calculus operators. In: Recent Advances in Applied Mathematics, Proceedings of the International Workshop Held at Kuwait University (Kuwait; May 4-7, 1996), pp. 441-450. Kuwait University, Department of Mathematics and Computer Science, Kuwait (1996)

27. Saigo, M.: A remark on integral operators involving the Gauss hypergeometric functions. Math. Rep. Kyushu Univ. 11, 135-143 (1977/78)

28. Saigo, M., Maeda, N.: More generalization of fractional calculus. In: Rusev, P., Dimovski, I., Kiryakova, V. (eds.) Transform Methods and Special Functions, Proceedings of the Second International Workshop Dedicated to the 100th Anniversary of the Birth of Nikola Obreschkoff (Varna; August 23-30, 1996), pp. 386-400. Bulgarian Academy of Sciences, Institute of Mathematics and Informatics, Sofia (1998)

29. Sneddon, I.N.: The Use of the Integral Transforms. Tata McGraw-Hill, New Delhi (1979)

30. Srivastava, H.M.: A contour integral involving Fox's H-function. Indian J. Math. 14, 1-6 (1972)

31. Srivastava, H.M., Chaudhry, M.A., Agarwal, R.P.: The incomplete Pochhammer symbols and their applications to hypergeometric and related functions. Integral Transforms Spec. Funct. 23, 659-683 (2012)

32. Srivastava, H.M., Karlsson, P.W.: Multiple Gaussian Hypergeometric Series. Halsted, New York (1985)

33. Srivastava, H.M., Manocha, H.L.: A Treatise on Generating Functions. Halsted, New York (1984)

34. Srivastava, H.M., Saxena, R.K.: Operators of fractional integration and their applications. Appl. Math. Comput. 118, 1-52 (2001)

35. Srivastava, H.M., Saxena, R.K., Parmar, R.K.: Some families of the incomplete $H$-functions and the incomplete $\bar{H}$-functions and associated integral transforms and operators of fractional calculus with applications. Russ. J. Math. Phys. 25(1), 116-138 (2018)

36. Srivastava, R.: Some properties of a family of incomplete hypergeometric functions. Russ. J. Math. Phys. 20, 121-128 (2013)

37. Srivastava, R., Agarwal, R., Jain, S.: A family of the incomplete hypergeometric functions and associated integral transform and fractional derivative formulas. Filomat 31, 125-140 (2017)

38. Srivastava, R., Cho, N.E.: Generating functions for a certain class of incomplete hypergeometric polynomials. Appl. Math. Comput. 219, 3219-3225 (2012) 
39. Suthar, D.L., Khan, A.M., Alaria, A., Purohit, S.D., Singh, J.: Extended Bessel-Maitland function and its properties pertaining to integral transforms and fractional calculus. AIMS Math. 5(2), 1400-1410 (2020)

40. Suthar, D.L., Purohit, S.D., Araci, S.: Solution of fractional kinetic equations associated with the (p, q)-Mathieu-type series. Discrete Dyn. Nat. Soc. 2020, 8645161 (2020)

41. Yang, X.-J.: General Fractional Derivatives: Theory, Methods and Applications. CRC Press, New York (2019)

42. Yang, X.-J., Abdel-Aty, M., Cattani, C.: A new general fractional-order derivative with Rabotnov fractional-exponential kernel applied to model the anomalous heat transfer. Therm. Sci. 23(3A), 1677-1681 (2019)

43. Yang, X.-J., Feng, Y.Y., Cattani, C., Inc, M.: Fundamental solutions of anomalous diffusion equations with the decay exponential kernel. Math. Methods Appl. Sci. 42(11), 4054-4060 (2019)

44. Yang, X.J., Gao, F., Ju, Y.: General Fractional Derivatives with Applications in Viscoelasticity. Academic Press, San Diego (2020)

45. Yang, X.-J., Gao, F., Ju, Y., Zhou, H.-W.: Fundamental solutions of the general fractional-order diffusion equations. Math. Methods Appl. Sci. 41(18), 9312-9320 (2018)

46. Yang, X.-J., Ragulskis, M., Taha, T.: A new general fractional-order derivative with Rabotnov fractional-exponential kernel. Therm. Sci. 23(6B), 3711-3718 (2019)

47. Yang, X.-J., Srivastava, H.M., Tenreiro Machado, J.A.: A new fractional derivative without singular kernel: application to the modelling of the steady heat flow. Therm. Sci. 20(2), 753-756 (2015)

48. Yang, X.-J., Tenreiro Machado, J.A.: A new fractal nonlinear Burgers' equation arising in the acoustic signals propagation. Math. Methods Appl. Sci. 42(18), 7539-7544 (2019)

\section{Submit your manuscript to a SpringerOpen ${ }^{\circ}$ journal and benefit from:}

- Convenient online submission

- Rigorous peer review

- Open access: articles freely available online

- High visibility within the field

- Retaining the copyright to your article

Submit your next manuscript at $\gg$ springeropen.com 